TRANSACTIONS OF THE

AMERICAN MATHEMATICAL SOCIETY

Volume 350, Number 11, November 1998, Pages 4593-4607

S 0002-9947(98)02312-5

\title{
THE DYNKIN-LAMPERTI ARC-SINE LAWS FOR MEASURE PRESERVING TRANSFORMATIONS
}

\author{
MAXIMILIAN THALER
}

\begin{abstract}
Arc-sine laws in the sense of renewal theory are proved for return time processes generated by transformations with infinite invariant measure on sets satisfying a type of Darling-Kac condition, and an application to real transformations with indifferent fixed points is discussed.
\end{abstract}

\section{INTRODUCTION}

The aim of the present paper is to contribute to the investigation of the probabilistic laws inherent in dynamical systems with infinite invariant measure. We deal in particular with the iterated maps on the unit interval with indifferent fixed points studied in [13], [14] and [16]. The first impulse for the considerations in this paper came from computer experiments with maps of this type ([15]).

The probabilistic laws considered are the arc-sine laws of renewal theory in the sense of E.B. Dynkin and J. Lamperti ([6], [9]). The underlying processes are determined by the successive visits to subsets of the state space under the iteration of a transformation with an infinite invariant measure. The lack of independence is compensated by J. Aaronson's uniform set condition introduced in [3]. Choosing a condition of this type has been suggested by J. Lamperti's condition (U) in [9] and the theorem in [16].

The paper is organized as follows. In section 2 we introduce the random variables to be studied and the necessary prerequisites from ergodic theory and probability theory. In section 3 we state and prove our version of the Dynkin-Lamperti theorem. In section 4 we discuss the application of the theorem to the class of one-dimensional maps referred to above.

\section{Preliminaries}

Let $(M, \mathcal{R}, \mu)$ be a $\sigma$-finite measure space, and let $T: M \rightarrow M$ be a conservative, ergodic, measure preserving transformation. Let $\mathcal{D}$ denote the set of probability measures on $\mathcal{R}$ absolutely continuous with respect to $\mu$. These measures represent the admissible initial distributions for the processes associated with the iteration of $T$. The symbol $\mathcal{D}$ will also be used for the set of the corresponding densities.

Received by the editors October 29, 1996.

1991 Mathematics Subject Classification. Primary 28D05, 60F05, 60K05.

(C)1998 American Mathematical Society 
We recall the notion of the wandering rate, which plays a key role in our context. Let $A \in \mathcal{R}$ be a set of positive finite measure, and let

$$
A_{n}=\bigcup_{k=0}^{n} T^{-k} A, \quad n \geq 0 .
$$

Then the (order of the) sequence $\left\{\mu\left(A_{n}\right)\right\}$ is called the wandering rate of $A$. From the probabilistic point of view it is essential to note that

$$
\mu\left(A_{n}\right)=\int_{A} \min \{\varphi, n+1\} d \mu(n \geq 0),
$$

where $\varphi$ is the first return time to the set $A$ (cf. [3], [14]).

The random variables to be studied in this paper arise in the following way.

Consider the process of successive vists to a fixed set $A \in \mathcal{R}$ of positive finite measure under the iteration of $T$, where the iterated point is chosen according to some distribution $\nu \in \mathcal{D}$. Inspecting the process at time $n$ leads to the observation of the variables

$$
\begin{aligned}
& Z_{n}(x):=\max \left\{k \leq n: T^{k}(x) \in A\right\}, \quad x \in A_{n}, \\
& Y_{n}(x):=\min \left\{k>n: T^{k}(x) \in A\right\}, \quad x \in M, \\
& V_{n}(x):=Y_{n}(x)-Z_{n}(x), \quad x \in A_{n} .
\end{aligned}
$$

According to the terminology of renewal theory, $n-Z_{n}$ is the spent waiting time, $Y_{n}-n$ the residual waiting time and $V_{n}$ the total waiting time, if the process is inspected at time $n$. For notational simplicity we suppress the dependence of the variables on the set $A$. Since $T$ is conservative and ergodic, for all $\nu \in \mathcal{D}$

$$
\lim _{n \rightarrow \infty} \nu\left(A_{n}\right)=1 \text {, and } \nu\left(\left\{Y_{n}<\infty\right\}\right)=1 \text { for all } n \geq 1 \text {. }
$$

It is convenient to extend the definition of $Z_{n}$ and $V_{n}$ to $M$ by putting $Z_{n}=0$ on $M \backslash A_{n}$. The way the extension is specified will not affect our statements, as is immediate if the process starts in $A$ with probability 1 .

We shall be interested in the asymptotic distributional behaviour of the random variables $Z_{n}, Y_{n}, V_{n}$. The type of convergence is made precise by the following notation. If $X_{n}: M \rightarrow[0, \infty](n \geq 1)$ is a sequence of measurable functions and $\xi$ is a random variable on $[0, \infty]$, we write

$$
X_{n} \stackrel{\nu}{\longrightarrow} \xi
$$

if the distribution of $X_{n}$ with respect to $\nu(\nu \in \mathcal{D})$ converges to the distribution of $\xi$ in the usual sense (see [1]).

The limiting distributions occurring in our context are the so called generalized arc-sine distributions and their relatives. For $0<\alpha<1$ we denote by $\zeta_{\alpha}$ a random variable on $[0,1]$ with density

$$
f_{\zeta_{\alpha}}(x)=\frac{\sin \pi \alpha}{\pi} \frac{1}{x^{1-\alpha}(1-x)^{\alpha}}, \quad 0<x<1 .
$$

Hence $\zeta_{\alpha}$ has the $B(\alpha, 1-\alpha)$ distribution, called the generalized arc-sine distribution. Continuous extension to the parameter interval $[0,1]$ yields $\zeta_{0}=0$ and $\zeta_{1}=1$. We recall that the moments of $\zeta_{\alpha}$ are given by

$$
E\left(\zeta_{\alpha}^{r}\right)=(-1)^{r}\left(\begin{array}{c}
-\alpha \\
r
\end{array}\right) \quad(r \geq 0, \alpha \in[0,1]) .
$$

In particular, $E\left(\zeta_{\alpha}\right)=\alpha$ for all $\alpha \in[0,1]$. 
The reciprocal $\zeta_{\alpha}^{-1}$ has the density

$$
f_{\zeta_{\alpha}^{-1}}(x)=\frac{\sin \pi \alpha}{\pi} \frac{1}{x(x-1)^{\alpha}}, \quad x>1,
$$

if $0<\alpha<1$. The continuous extension is given by $\zeta_{0}^{-1}=\infty$ and $\zeta_{1}^{-1}=1$. (Throughout the paper we will follow the conventions $\frac{1}{0}=\infty, \frac{1}{\infty}=0$.)

Finally, for $0<\alpha<1$ let $\eta_{\alpha}$ denote a random variable on $[0, \infty)$ with density

$$
f_{\eta_{\alpha}}(x)=\frac{\sin \pi \alpha}{\pi} \frac{1-(\max \{1-x, 0\})^{\alpha}}{x^{1+\alpha}}, \quad x>0,
$$

extended by $\eta_{0}=\infty, \eta_{1}=0$. We note that $P\left(\eta_{\alpha}>1\right)=\tau(\alpha)$ for all $\alpha \in[0,1]$, where

$$
\tau(\alpha)=\frac{\sin \pi \alpha}{\pi \alpha}, \quad 0<\alpha \leq 1, \tau(0)=1 .
$$

As mentioned in the introduction we adopt the concept of uniform sets from [3]. It is an appropriate type of Darling-Kac condition for our purpose (cf. also [5], [9], $[2])$.

Let $\hat{T}: L_{1}(\mu) \rightarrow L_{1}(\mu)$ denote the operator dual to $g \mapsto g \circ T, g \in L_{\infty}(\mu)$. A set $A \in \mathcal{R}, 0<\mu(A)<\infty$, is called uniform for $f \in \mathcal{D}$, if there exists a sequence $\left\{a_{n}\right\}$ of positive real numbers such that

$$
\left(\frac{1}{a_{n}} \sum_{k=0}^{n-1} \hat{T}^{k} f\right)_{\left.\right|_{A}} \longrightarrow 1 \quad \text { in } \quad L_{\infty}\left(\mu_{\left.\right|_{A \cap \mathcal{R}}}\right) .
$$

Integration shows that $\mu(A) a_{n} \sim \sum_{k=0}^{n-1} \nu\left(T^{-k} A\right)(n \rightarrow \infty)$, where $\nu$ is the probability measure with density $f$. $A$ is called a uniform set, if it is uniform for some $f \in \mathcal{D}$.

Using arguments as in the proof of Proposition 3.7.5 in [3] one can see that $T$ admits uniform sets if and only if it is pointwise dual ergodic. A slightly refined reasoning shows that, if $\left\{\frac{1}{a_{n}} \sum_{k=0}^{n-1} \hat{T}^{k} f\right\}$ converges to a positive finite limit function $g$ on a set of positive measure, then $g$ is constant thereon. Hence it is no restriction admitting only constant limits in the definition. Concerning the relationship to condition (U) in [9] see the remark at the end of the next section, where we will also review the arguments just referred to in terms of Laplace transforms.

For the background on Karamata's theory of regular variation needed in the sequel we refer to [4] and [7]. In particular, we shall repeatedly make use of Lamperti's criterion for regular variation $([8])$ :

Let $g:(0, \beta) \rightarrow(0, \infty)(\beta>0)$ be differentiable and convex. Then $g$ is regularly varying for $x \rightarrow 0$ with index $\rho(\rho \in \mathbf{R})$, if and only if

$$
\lim _{x \rightarrow 0} \frac{x g^{\prime}(x)}{g(x)}=\rho .
$$

The criterion extends to rapid variation, i.e. to the cases $\rho= \pm \infty$.

\section{THE LIMIT THEOREM}

With the preparations in section 2 we can now state our main result.

Theorem 1. Let $A \in \mathcal{R}, 0<\mu(A)<\infty$, be a uniform set, and let $\alpha$ be in $[0,1]$. Then the following assertions are equivalent.

(1) The wandering rate of $A$ is regularly varying with index $1-\alpha$.

(2) $\frac{1}{n} Z_{n} \stackrel{\nu}{\longrightarrow} \zeta_{\alpha}$ for all $\nu \in \mathcal{D}$. 
(3) $\frac{1}{n} Y_{n} \stackrel{\nu}{\longrightarrow} \zeta_{\alpha}^{-1}$ for all $\nu \in \mathcal{D}$.

(4) $\frac{1}{n} V_{n} \stackrel{\nu}{\longrightarrow} \eta_{\alpha}$ for all $\nu \in \mathcal{D}$.

(2), (3) and (4) may be replaced by the assertion that the respective sequence converges in distribution to some random variable on $[0, \infty]$ with respect to some $\nu \in \mathcal{D}$. If the limiting random variables are $\zeta, \xi, \eta$ respectively, the parameter $\alpha$ is recovered by the relations $E(\zeta)=\alpha, E\left(\xi^{-1}\right)=\alpha$, and $P(\eta>1)=\tau(\alpha)$.

We note first that parts of the implications of Theorem 1 are immediate from the following identities (see [6]).

For $1 \leq k \leq n$,

$$
\left\{Z_{n} \leq k\right\}=\left\{Y_{k}>n\right\},
$$

and, more generally, for $1 \leq k \leq n \leq m$,

$$
\left\{Z_{n} \leq k, Y_{n}>m\right\}=\left\{Z_{m} \leq k\right\} .
$$

The first identity shows that the statements on $\left\{Y_{n}\right\}$ are equivalent to those on $\left\{Z_{n}\right\}$. The second identity shows that (2) implies (4). For, if (2) holds with $0<\alpha<$ 1 , then due to the second identity the joint distribution of $\left(\frac{1}{n} Z_{n}, \frac{1}{n} Y_{n}\right)$ converges to the distribution with density

$$
-\frac{\partial}{\partial y} f_{\zeta_{\alpha}}\left(\frac{x}{y}\right) \frac{1}{y}, \quad 0<x<1<y,
$$

and a standard computation then yields (4). The implication is obvious if $\alpha=0$ or $\alpha=1$.

The first of the two lemmas to follow together with a proposition of J. Aaronson's will show that it suffices to consider one specific probability measure in $\mathcal{D}$.

Lemma 1. Let $A \in \mathcal{R}$ be a set of positive finite measure, and let $\left\{X_{n}\right\}$ be any of the sequences $\left\{Z_{n}\right\},\left\{Y_{n}\right\},\left\{V_{n}\right\}$. Then

$$
\frac{1}{n}\left(X_{n}-X_{n} \circ T\right) \stackrel{\nu}{\longrightarrow} 0 \quad \text { for all } \nu \in \mathcal{D} .
$$

Proof. Let $\varphi$ denote the time of the first visit to the set $A$ after time 0 . Let $\varepsilon>0$ be given, and let

$$
K_{\varepsilon, n}:=\left\{\varphi \leq n, \frac{1}{n}\left|Z_{n}-Z_{n} \circ T\right| \geq \varepsilon\right\} .
$$

Choose $n$ so large that $\frac{1}{n}<\varepsilon$. Since

$$
\left|Z_{n}(x)-Z_{n}(T x)\right|=\left\{\begin{array}{cl}
1, & x \in\{\varphi \leq n\} \cap T^{-(n+1)} A^{c}, \\
n-Z_{n}(x), & x \in T^{-(n+1)} A,
\end{array}\right.
$$

we have

$$
\begin{aligned}
K_{\varepsilon, n} & =\bigcup_{n \varepsilon \leq k \leq n-1}\left\{Z_{n}=n-k\right\} \cap T^{-(n+1)} A \\
& =\bigcup_{1+n \varepsilon \leq k \leq n} T^{-(n-k+1)}(A \cap\{\varphi=k\}) .
\end{aligned}
$$

Using the invariance of $\mu$ we obtain

$$
\mu\left(K_{\varepsilon, n}\right)=\sum_{1+n \varepsilon \leq k \leq n} \mu(A \cap\{\varphi=k\}) \leq \mu(A \cap\{\varphi \geq 1+n \varepsilon\}),
$$


and therefore

$$
\lim _{n \rightarrow \infty} \mu\left(K_{\varepsilon, n}\right)=0 \text {. }
$$

This implies $\lim _{n \rightarrow \infty} \nu\left(K_{\varepsilon, n}\right)=0$ for all $\nu \in \mathcal{D}$, and hence finishes the proof for the sequence $\left\{Z_{n}\right\}$, since $\lim _{n \rightarrow \infty} \nu(\{\varphi>n\})=0$ anyway.

To verify the assertion for $\left\{Y_{n}\right\}$, note that

$$
\left|Y_{n}(x)-Y_{n}(T x)\right|=\left\{\begin{array}{cl}
1, & x \in\left\{Y_{n}<\infty\right\} \cap T^{-(n+1)} A^{c}, \\
\varphi\left(T^{n+1} x\right)-1, & x \in T^{-(n+1)} A,
\end{array}\right.
$$

and hence, if $n>\frac{1}{\varepsilon}$,

$$
\left\{Y_{n}<\infty, \frac{1}{n}\left|Y_{n}-Y_{n} \circ T\right| \geq \varepsilon\right\}=T^{-(n+1)}(A \cap\{\varphi \geq 1+n \varepsilon\}) .
$$

Again we complete the argument using the invariance of $\mu$. The sequence $\left\{V_{n}\right\}$ inherits the asserted property from $\left\{Z_{n}\right\}$ and $\left\{Y_{n}\right\}$.

As an immediate consequence of Lemma 1 , if $A \in \mathcal{R}$ is a set of positive finite measure and $\left\{X_{n}\right\}$ is any of the sequences in question,

$$
g\left(\frac{1}{n} X_{n}\right)-g\left(\frac{1}{n} X_{n} \circ T\right) \stackrel{\nu}{\longrightarrow} 0
$$

for all $g \in C([0, \infty])$ and all $\nu \in \mathcal{D}$. Since the convergence is dominated this is equivalent to

$$
g\left(\frac{1}{n} X_{n}\right)-g\left(\frac{1}{n} X_{n} \circ T\right) \stackrel{w^{*}}{\longrightarrow} 0
$$

in $L_{\infty}(\mu)$ for all $g \in C([0, \infty])$. The reasoning in the proof of Proposition 0 in [1] ( $\mathrm{cf}$. also [18]) now shows that for every subsequence of $\mathbf{N}$ there exists a subsequence $\left\{n_{k}\right\}$ and a probability measure $\tau$ on $[0, \infty]$ such that the sequence $\left\{\frac{1}{n_{k}} X_{n_{k}}\right\}$ converges in distribution to $\tau$ with respect to every $\nu \in \mathcal{D}$. Thus $\frac{1}{n} X_{n} \stackrel{\nu}{\longrightarrow} \xi$ for at least one $\nu \in \mathcal{D}$ implies $\frac{1}{n} X_{n} \stackrel{\nu}{\longrightarrow} \xi$ for all $\nu \in \mathcal{D}$.

As in [9] we shall deduce assertion (2) in Theorem 1 from condition (1) by means of the method of moments. Our procedure is based on a direct generalization of the asymptotic renewal equation in [2] and [3] (see Lemma 2 below) and on the observation that for each $r \in \mathbf{N}$ the sequence of $r$-th moments of $\left\{Z_{n}\right\}$ is increasing by the very definition of the variables $Z_{n}$.

Given a set $A \in \mathcal{R}$ of positive finite measure let

$$
q_{n}=\frac{\mu(A \cap\{\varphi>n\})}{\mu(A)}, \quad n \geq 0,
$$

and let the Laplace transforms $Q(s), U(s), H_{r}(s)\left(s>0, r \in \mathbf{N}_{0}\right)$ be defined by

$$
\begin{aligned}
Q(s) & =\sum_{n=0}^{\infty} q_{n} e^{-n s}, \\
U(s) & =\sum_{n=0}^{\infty} \nu\left(T^{-n} A\right) e^{-n s}, \\
H_{r}(s) & =\sum_{n=0}^{\infty}\left(\int_{A_{n}}\left(n-Z_{n}\right)^{r} d \nu\right) e^{-n s} .
\end{aligned}
$$


Lemma 2. If $A \in \mathcal{R}, 0<\mu(A)<\infty$, is uniform for $f \in \mathcal{D}$, and $\nu$ is the probability measure with density $f$, then

$$
H_{r}(s) \sim(-1)^{r} U(s) Q^{(r)}(s) \sim \frac{1}{s} \frac{(-1)^{r} Q^{(r)}(s)}{Q(s)} \quad(s \rightarrow 0)
$$

for all $r \in \mathbf{N}_{0}$.

Proof. For $r, n \geq 0$,

$$
\begin{aligned}
\int_{A_{n}}\left(n-Z_{n}\right)^{r} d \nu & =\sum_{k=0}^{n}(n-k)^{r} \nu\left(A_{n} \cap\left\{Z_{n}=k\right\}\right) \\
& =\sum_{k=0}^{n}(n-k)^{r} \nu\left(T^{-k}(A \cap\{\varphi>n-k\})\right) \\
& =\int_{A} \sum_{k=0}^{n} \hat{T}^{k} f(n-k)^{r} 1_{A \cap\{\varphi>n-k\}} d \mu .
\end{aligned}
$$

Hence,

$$
H_{r}(s)=\int_{A}\left(\sum_{n=0}^{\infty} \hat{T}^{n} f e^{-n s}\right)\left(\sum_{n=0}^{\infty} n^{r} 1_{A \cap\{\varphi>n\}} e^{-n s}\right) d \mu .
$$

Since $A$ is uniform for $f$, the identity

$$
\left(\sum_{n=0}^{\infty} \hat{T}^{n} f e^{-n s}\right) / U(s)=\left(\sum_{n=1}^{\infty}\left(\sum_{k=0}^{n-1} \hat{T}^{k} f\right) e^{-n s}\right) /\left(\sum_{n=1}^{\infty}\left(\sum_{k=0}^{n-1} \nu\left(T^{-k} A\right)\right) e^{-n s}\right)
$$

yields

$$
\left(\sum_{n=0}^{\infty}\left(\hat{T}^{n} f\right)_{\left.\right|_{A}} e^{-n s}\right) / U(s) \stackrel{s \rightarrow 0}{\longrightarrow} 1 / \mu(A) \quad \text { in } L_{\infty}\left(\mu_{\left.\right|_{A \cap \mathcal{R}}}\right) .
$$

Therefore the first relation follows from the above representation of $H_{r}(s)$.

Observing that $\lim _{n \rightarrow \infty} \nu\left(A_{n}\right)=1$ we obtain for $r=0$

$$
U(s) Q(s) \sim \frac{1}{s}(s \rightarrow 0),
$$

which implies the second relation.

Now we complete the

Proof of Theorem 1. Suppose $A$ is uniform for $f$, and let $Q$ and $H_{r}$ be defined with respect to $A$, where $\nu$ denotes the probability measure with density $f$. First we prove the implication $(1) \Rightarrow(2)$. Since

$$
\mu\left(A_{n}\right)=\sum_{k=0}^{n} \mu(A \cap\{\varphi>k\})=\mu(A) \sum_{k=0}^{n} q_{k}, \quad n \geq 1,
$$

condition (1) together with Karamata's Tauberian Theorem (cf. [4], [7]) yields

$$
Q(s) \sim\left(\frac{1}{s}\right)^{1-\alpha} L\left(\frac{1}{s}\right) \quad(s \rightarrow 0),
$$

where $L$ is slowly varying at infinity. By the Lamperti criterion resp. the Monotone Density Theorem of the theory of regular variation we may differentiate treating $L$ as a constant. This gives

$$
Q^{(r)}(s) \sim r !\left(\begin{array}{c}
\alpha-1 \\
r
\end{array}\right)\left(\frac{1}{s}\right)^{r+1-\alpha} L\left(\frac{1}{s}\right)(s \rightarrow 0) \text { for all } r \geq 0 .
$$


With the notation

$$
m_{\beta, r}:=E\left(\zeta_{\beta}^{r}\right)
$$

we then obtain from Lemma 2

$$
H_{r}(s) \sim r ! m_{1-\alpha, r}\left(\frac{1}{s}\right)^{r+1}(s \rightarrow 0),
$$

and hence by differentiation

$$
H_{j}^{(r-j)}(s) \sim(-1)^{r-j} r ! m_{1-\alpha, j}\left(\frac{1}{s}\right)^{r+1}(s \rightarrow 0) \quad \text { for } 0 \leq j \leq r, r \geq 0 .
$$

Now we pass to the transforms

$$
G_{r}(s)=\sum_{n=0}^{\infty}\left(\int_{M} Z_{n}^{r} d \nu\right) e^{-n s} \quad(s>0, r \geq 1)
$$

by means of the identities

$$
\begin{aligned}
G_{r}(s) & =(-1)^{r} \sum_{j=0}^{r}\left(\begin{array}{l}
r \\
j
\end{array}\right) H_{j}^{(r-j)}(s), \text { and } \\
m_{\alpha, r} & =\sum_{j=0}^{r}\left(\begin{array}{l}
r \\
j
\end{array}\right)(-1)^{j} m_{1-\alpha, j} .
\end{aligned}
$$

The first is easily verified using

$$
Z_{n}^{r}=\sum_{j=0}^{r}\left(\begin{array}{l}
r \\
j
\end{array}\right)(-1)^{j} n^{r-j}\left(n-Z_{n}\right)^{j} ;
$$

the second is a consequence of the fact that $\zeta_{\alpha}$ and $1-\zeta_{1-\alpha}$ have the same distribution. From the asymptotics of the functions $H_{r}^{(r-j)}$ we then obtain

$$
G_{r}(s) \sim r ! m_{\alpha, r}\left(\frac{1}{s}\right)^{r+1}(s \rightarrow 0),
$$

and Karamata's Tauberian Theorem yields

$$
\int_{M} Z_{n}^{r} d \nu \sim m_{\alpha, r} n^{r}(n \rightarrow \infty),
$$

proving assertion (2). (We note that in the classical case one would work directly with the transforms $G_{r}$, interchanging the roles of $Q$ and $U$ in Lemma 2.)

Assume conversely that $\frac{1}{n} Z_{n} \stackrel{\nu}{\longrightarrow} \zeta$ for some random variable $\zeta$ on $[0,1]$ with $E(\zeta)=\alpha$. Then the first moments of $\left\{\frac{1}{n} Z_{n}\right\}$ converge to $\alpha$, and we know from [9] that this suffices to obtain condition (1). In fact,

$$
\lim _{n \rightarrow \infty} \frac{1}{n} \int_{A_{n}}\left(n-Z_{n}\right) d \nu=1-\alpha
$$

together with Lemma 2 yields

$$
\lim _{s \rightarrow 0} \frac{s Q^{\prime}(s)}{Q(s)}=-(1-\alpha)
$$

which means that $Q(s)$ is regularly varying for $s \rightarrow 0$ with index $-(1-\alpha)$.

It remains to prove that $\frac{1}{n} V_{n} \stackrel{\nu}{\longrightarrow} \eta$ for some random variable $\eta$ on $[0, \infty]$ with $P(\eta>1)=\tau(\alpha)$ implies (1). 
For $x \geq 1$,

$$
\begin{aligned}
\nu\left(\left\{\frac{1}{n} V_{n}>x\right\} \cap A_{n}\right) & =\sum_{k=0}^{n} \nu\left(T^{-k}(A \cap\{\varphi>n x\})\right) \\
& =\int_{A}\left(\sum_{k=0}^{n} \hat{T}^{k} f\right) 1_{A \cap\{\varphi>n x\}} d \mu .
\end{aligned}
$$

Since $A$ is uniform for $f$,

$$
\int_{A}\left(\sum_{k=0}^{n} \hat{T}^{k} f\right) 1_{A \cap\{\varphi>n x\}} d \mu \sim a_{n} \mu(A \cap\{\varphi>n x\})(n \rightarrow \infty),
$$

where $\left\{a_{n}\right\}$ is the associated normalizing sequence. Hence

$$
\lim _{n \rightarrow \infty} a_{n} \mu(A \cap\{\varphi>n x\})=P(\eta>x)
$$

if $x$ is a continuity point of the limiting distribution.

First suppose $P(\eta>1)>0$. Then, using an argument as in the proof of Lemma 3 in VIII.8 of [7], we see that

$$
\lim _{t \rightarrow \infty} \frac{\mu(A \cap\{\varphi>x t\})}{\mu(A \cap\{\varphi>t\})}
$$

exists and is positive on an $x$-set of positive measure, and the Characterization Theorem for regular variation shows that $\{\mu(A \cap\{\varphi>n\})\}$ is regularly varying. Therefore $\left\{\mu\left(A_{n}\right)\right\}$ is regularly varying. Denote the index by $1-\gamma(\gamma \in[0,1])$. Since we already know that this implies $\eta=\eta_{\gamma}$, we conclude that $\tau(\gamma)=\tau(\alpha)$, i.e. $\gamma=\alpha$.

If $P(\eta>1)=0$,

$$
\lim _{n \rightarrow \infty} a_{n} \mu(A \cap\{\varphi>n x\})=0 \quad \text { for all } x>1 .
$$

From the estimate

$$
\sum_{k=0}^{n} \nu\left(A_{k}\right) \leq \int_{A}\left(\sum_{k=0}^{n} \hat{T}^{k} f\right)\left(\sum_{k=0}^{n} 1_{A \cap\{\varphi>k\}}\right) d \mu,
$$

contained in the proof of Lemma 2, it follows that

$$
\liminf _{n \rightarrow \infty} \frac{a_{n} \mu\left(A_{n}\right)}{n} \geq 1
$$

Therefore, choosing $x=2$ in the above relation we obtain

$$
\lim _{n \rightarrow \infty} \frac{n \mu(A \cap\{\varphi>2 n\})}{\mu\left(A_{n}\right)}=0,
$$

and hence, taking into account the monotonicity of the sequences involved,

$$
\lim _{n \rightarrow \infty} \frac{n \mu(A \cap\{\varphi>n\})}{\mu\left(A_{n}\right)}=0 .
$$

By the standard criterion for regular variation of sequences, $\left\{\mu\left(A_{n}\right)\right\}$ is slowly varying, in accordance with $P(\eta>1)=\tau(1)$. This completes the proof of Theorem 1. 
Remark. If $A \in \mathcal{R}, 0<\mu(A)<\infty$, is uniform for $f$, then

$$
\left(\sum_{n=0}^{\infty}\left(\hat{T}^{n} f\right)_{\left.\right|_{A}} e^{-n s}\right) / U(s) \stackrel{s \rightarrow 0}{\longrightarrow} 1 / \mu(A) \quad \text { in } L_{\infty}\left(\mu_{\left.\right|_{A \cap \mathcal{R}}}\right),
$$

as stated in the proof of Lemma 2. This relation is equivalent to condition (U) in [9], if we translate it into our context as follows.

A pair $(A, f)$ with $A \in \mathcal{R}, 0<\mu(A)<\infty$, and $f \in \mathcal{D}$ satisfies condition (U) if there exists a probability measure $\kappa$ on $A \cap \mathcal{R}$ and a function $r(E, s)$ such that

$$
\begin{aligned}
& \sum_{n=0}^{\infty} \nu\left(T^{-n} E\right) e^{-n s}=\kappa(E)(U(s)+r(E, s)) \quad(E \in A \cap \mathcal{R}, s>0) \\
& \text { and } \sup _{E \in A \cap \mathcal{R}} \frac{|r(E, s)|}{U(s)} \stackrel{s \rightarrow 0}{\longrightarrow} 0,
\end{aligned}
$$

where $\nu$ denotes the probability measure with density $f$ and $U(s)$ is defined as before. We may assume $r(E, s)=0$ if $\kappa(E)=0$.

It is immediate that $(*)$ implies (U) with $\kappa(E)=\mu(E) / \mu(A), E \in A \cap \mathcal{R}$. We show conversely that this is the only possible choice for $\kappa$, proving the asserted equivalence.

Assume that condition (U) holds. Since $\nu\left(T^{-n} E\right)=0$ for all $n \geq 0$, if and only if $\mu(E)=0, \kappa$ and $\mu$ are equivalent on $A \cap \mathcal{R}$. Let $g=d \kappa / d \mu$. Then,

$$
\begin{aligned}
\sup _{E \in A \cap \mathcal{R}} \frac{|r(E, s)|}{U(s)} & =\sup _{\substack{E \in A \cap \mathcal{R} \\
\kappa(E)>0}} \frac{1}{\kappa(E)}\left|\int_{E}\left(\left(\sum_{n=0}^{\infty} \hat{T}^{n} f e^{-n s}\right) /(g U(s))-1\right) d \kappa\right| \\
& =\left\|\left(\sum_{n=0}^{\infty} \hat{T}^{n} f e^{-n s}\right) /(g U(s))-1\right\|_{L_{\infty}\left(\mu_{\mid A \cap \mathcal{R}}\right)}(s>0),
\end{aligned}
$$

hence

$$
\left(\sum_{n=0}^{\infty}\left(\hat{T}^{n} f\right)_{\left.\right|_{A}} e^{-n s}\right) /(g U(s)) \stackrel{s \rightarrow 0}{\longrightarrow} 1 \quad \text { in } L_{\infty}\left(\mu_{\left.\right|_{A \cap \mathcal{R}}}\right) .
$$

To see that $g$ is constant we use arguments as in the proof of Proposition 3.7.5 in [3]. Let $a, b \geq 0$ and $0<s_{0}<1$ be given so that the set

$$
B:=\left\{a U(s) \leq \sum_{n=0}^{\infty} \hat{T}^{n} f e^{-n s} \leq b U(s) \text { for } 0<s<s_{0}\right\}
$$

has positive measure. Let $\varphi_{B}$ denote the time of the first visit to $B$ after time 0 , and let $B_{n}=\bigcup_{k=0}^{n} T^{-k} B, n \geq 0$. By means of the decomposition

$$
B_{n}=\bigcup_{k=0}^{n} T^{-(n-k)} D_{k}(n \geq 0), \text { where } D_{k}=B \cap\left\{\varphi_{B}>k\right\}(k \geq 0),
$$

we obtain the following analogue to the basic formula in the proof of the proposition mentioned above:

$$
\sum_{n=0}^{\infty} \hat{T}^{n} f e^{-n s}=\sum_{n=0}^{\infty} \hat{T}^{n}\left(1_{D_{n}} \sum_{k=0}^{\infty} \hat{T}^{k} f e^{-k s}\right) e^{-n s}+\sum_{n=0}^{\infty} \hat{T}^{n}\left(f \cdot 1_{M \backslash B_{n}}\right) e^{-n s}
$$


$(s>0)$. The operator $\hat{T}$ being positive the formula yields

$$
\begin{aligned}
a \sum_{n=0}^{\infty} \hat{T}^{n} 1_{D_{n}} e^{-n s} & \leq\left(\sum_{n=0}^{\infty} \hat{T}^{n} f e^{-n s}\right) / U(s) \\
& \leq b \sum_{n=0}^{\infty} \hat{T}^{n} 1_{D_{n}}+\left(\sum_{n=0}^{\infty} \hat{T}^{n}\left(f \cdot 1_{M \backslash B_{n}}\right)\right) / U(s)
\end{aligned}
$$

(a.e.) on $M$ for $0<s<s_{0}$. Furthermore, integration over the sets $B$ and $B^{c} \cap$ $\left\{\varphi_{B}=m\right\}(m \geq 1)$ shows that

$$
\sum_{n=0}^{\infty} \hat{T}^{n}\left(f .1_{M \backslash B_{n}}\right)<\infty \quad \text { a.e. on } M .
$$

Therefore, taking into account that $\sum_{n=0}^{\infty} \hat{T}^{n} 1_{D_{n}}=1$ a.e. and $\lim _{s \rightarrow 0} U(s)=\infty$, we get

$$
a \leq \liminf _{s \rightarrow 0}\left(\sum_{n=0}^{\infty} \hat{T}^{n} f e^{-n s}\right) / U(s) \leq \limsup _{s \rightarrow 0}\left(\sum_{n=0}^{\infty} \hat{T}^{n} f e^{-n s}\right) / U(s) \leq b
$$

a.e. on $M$. This argument shows that

$$
\lim _{s \rightarrow 0}\left(\sum_{n=0}^{\infty} \hat{T}^{n} f e^{-n s}\right) / U(s)=c \quad \text { a.e. on } M
$$

for some positive constant $c$. Hence $g=1 / \mu(A)$ on $A$, and $(*)$ holds.

\section{Application to MAPS With indiffEREnT FiXED POINTS}

To illustrate the conditions in Theorem 1 we consider the class of piecewise monotone transformations $T:[0,1] \rightarrow[0,1]$ with indifferent fixed points studied in [13], [14] and [16]. Examples of this type appear in different fields. In statistical physics they serve as models to study basic features of the phenomenon of intermittency in the sense of [10].

Let $\{B(k): k \in I\}$ be a family of pairwise disjoint subintervals of $[0,1]$ with $\lambda\left(\bigcup_{k \in I} B(k)\right)=1$ ( $\lambda$ denoting the Lebesgue measure on the Borel $\sigma$-field on $[0,1])$, and let $T:[0,1] \rightarrow[0,1]$ satisfy the following conditions.

1. For all $k \in I, T_{\left.\right|_{B(k)}}$ is twice differentiable and $\overline{T B(k)}=[0,1]$.

For a non-empty finite set $J \subseteq I$ the interval $B(j), j \in J$, contains a fixed point $x_{j}$ with $T^{\prime}\left(x_{j}\right)=1$.

2. $\left|T^{\prime}\right| \geq \rho(\varepsilon)>1$ on $\bigcup_{k \in I} B(k) \backslash \bigcup_{j \in J}\left(x_{j}-\varepsilon, x_{j}+\varepsilon\right)$ for each $\varepsilon>0$.

3. For $j \in J, T^{\prime}$ is decreasing on $\left(x_{j}-\eta, x_{j}\right) \cap B(j)$ and increasing on $\left(x_{j}, x_{j}+\right.$ $\eta) \cap B(j)$ for some $\eta>0$.

4. $T^{\prime \prime} /\left(T^{\prime}\right)^{2}$ is bounded on $\bigcup_{k \in I} B(k)$.

As proved in [13] and [14], $T$ fulfills the basic conditions of section 2 with respect to the (unique) invariant measure $\mu$ equivalent to $\lambda$. The density $d \mu / d \lambda$ has a version $h$ of the form

$$
h(x)=h_{0}(x) \prod_{j \in J} \frac{x-x_{j}}{x-f_{j}(x)}, x \in[0,1] \backslash\left\{x_{j}: j \in J\right\},
$$

where $f_{j}$ denotes the $C^{1}$-extension of $\left(T_{\left.\right|_{B(j)}}\right)^{-1}$ to $[0,1]$, and $h_{0}$ is continuous and positive on $[0,1]$. 
Let $E(T)$ denote the class of Borel subsets of $[0,1]$ with positive measure which are bounded away from the indifferent fixed points. The sets in $E(T)$ are natural candidates for which Theorem 1 may be expected to hold. Since

$$
\hat{T} f=\frac{1}{h} P(f h) \quad\left(f \in L_{1}(\mu)\right),
$$

where $P$ is the Perron-Frobenius operator of $T$ with respect to $\lambda$, the theorem in [16] implies that $A$ is uniform for $f$ for all $A \in E(T)$ and all $f \in \mathcal{D}$ for which $f h$ is Riemann-integrable on $[0,1]$. Hence all sets in $E(T)$ have the required Darling-Kac property. Furthermore, by Theorem 3 in [14],

$$
\mu\left(A_{n}\right) \sim \mu\left(B_{n}\right)(n \rightarrow \infty) \text { for all sets } A, B \in E(T) .
$$

In order to guarantee regular variation of these sequences we add the following condition.

5. For $j \in J, T x-x$ varies regularly (rapidly) for $x \rightarrow x_{j}^{+}$with index $1+p_{j}^{+}$(if $x_{j}<1$ ), and $x-T x$ varies regularly (rapidly) for $x \rightarrow x_{j}^{-}$with index $1+p_{j}^{-}$ (if $x_{j}>0$ ).

Due to condition 4 the extended real numbers $p_{j}^{+}, p_{j}^{-}$belong to $[1, \infty]$. We recall that rapid variation formally means regular variation with infinite index.

Condition 5 clearly holds with $p_{j}^{+}=p_{j}^{-}=p_{j}$, if the local behaviour of $T$ at $x_{j}, j \in J$, is of the form

$$
T(x)=x \pm a_{j}\left|x-x_{j}\right|^{1+p_{j}}+o\left(\left|x-x_{j}\right|^{1+p_{j}}\right)\left(x \rightarrow x_{j}\right)
$$

with $a_{j}>0,1 \leq p_{j}<\infty$.

Now we can state the desired result.

Theorem 2. Let $T:[0,1] \rightarrow[0,1]$ satisfy the conditions $1-5$, and let

$$
p:=\max \left\{p_{j}^{+}, p_{j}^{-}: j \in J\right\} .
$$

Then the Dynkin-Lamperti arc-sine laws hold with $\alpha=\frac{1}{p}$ for all sets $A$ in $E(T)$.

The missing link for the proof of Theorem 2 is provided by the following lemma which complements Lemma 2 in [14] (see also [12]). Following the notation in [4] we write $f \in R_{\rho}\left(a^{+}\right)$, if $f$ is regularly (rapidly) varying for $x \rightarrow a^{+}$with index $\rho$ and $\left\{a_{n}\right\} \in R_{\rho}$, if $\left\{a_{n}\right\}$ is a regularly varying sequence with index $\rho$.

Lemma 3. Let $f:[0, \eta] \longrightarrow \mathbf{R}(\eta>0)$ be differentiable and concave satisfying $0<f(x)<x, 0<x \leq \eta$. Let $a_{n}=f^{n}\left(a_{0}\right)(n \geq 0)$ for some $a_{0} \in(0, \eta]$, and let $r(x)=x-f(x)$. Then the following assertions hold.

(a) For all $\rho \in[0,1]$,

$$
\left\{\sum_{k=0}^{n} a_{k}\right\} \in R_{\rho}, \text { if and only if } \lim _{x \rightarrow 0}\left(x \int_{x}^{\eta} \frac{d y}{r(y)}\right) /\left(\int_{x}^{\eta} \frac{y}{r(y)} d y\right)=\rho .
$$

(b) If $r \in R_{1+p}\left(0^{+}\right)$with $1 \leq p \leq \infty$, then $\left\{\sum_{k=0}^{n} a_{k}\right\} \in R_{1-\frac{1}{p}}$.

$$
\text { If } 1<p<\infty \text {, the converse also holds. }
$$


Proof. (a) From the theory of regularly varying sequences we know that

$$
\left\{\sum_{k=0}^{n} a_{k}\right\} \in R_{\rho}, \text { if and only if } \lim _{n \rightarrow \infty}\left(n a_{n}\right) /\left(\sum_{k=0}^{n} a_{k}\right)=\rho .
$$

Let

$$
b(x):=\int_{x}^{\eta} \frac{d y}{r(y)}, \quad c(x):=\int_{x}^{\eta} \frac{y}{r(y)} d y \quad(0 \leq x \leq \eta) .
$$

Assume first $c(0)=\infty$. Then necessarily $f^{\prime}(0)=1$, and hence by Lemma 2 in [14],

$$
\begin{aligned}
& b\left(a_{n}\right) \sim n \quad(n \rightarrow \infty), \quad \text { and } \\
& c\left(a_{n}\right) \sim \sum_{k=0}^{n} a_{k} \quad(n \rightarrow \infty) .
\end{aligned}
$$

Therefore,

$$
\lim _{n \rightarrow \infty}\left(n a_{n}\right) /\left(\sum_{k=0}^{n} a_{k}\right)=\rho, \text { if and only if } \lim _{n \rightarrow \infty} a_{n} b\left(a_{n}\right) / c\left(a_{n}\right)=\rho .
$$

Since $b\left(a_{n}\right) \sim b\left(a_{n+1}\right)(n \rightarrow \infty)$ and $b(x), c(x)$ are monotone it is readily seen that the right hand side can be replaced by the corresponding continuous limit.

If $c(0)<\infty, \sum_{k=0}^{\infty} a_{k}$ converges. This is obviously true if $f^{\prime}(0)<1$, and is part of the lemma referred to above if $f^{\prime}(0)=1$. Since $c(0)<\infty$ implies $\lim _{x \rightarrow 0} x \int_{x}^{\eta} \frac{d y}{r(y)}=$ 0 , both sides in (a) hold with $\rho=0$.

(b) Assume $r \in R_{1+p}\left(0^{+}\right), p \in[1, \infty]$. Noting that $r$ is convex we can apply Lamperti's criterion to get

$$
\lim _{x \rightarrow 0} \frac{x r^{\prime}(x)}{r(x)}=1+p .
$$

Let $b(x), c(x)$ be as above. Integration by parts yields the identity

$$
c(x)=x b(x)+\int_{x}^{\eta} b(y) d y .
$$

If $c(0)=\infty$, we apply l'Hospital's rule twice to obtain

$$
\begin{aligned}
\lim _{x \rightarrow 0}\left(\int_{x}^{\eta} b(y) d y\right) / c(x) & =\lim _{x \rightarrow 0} b(x) /\left(\frac{x}{r(x)}\right) \\
& =\lim _{x \rightarrow 0} 1 /\left(\frac{x r^{\prime}(x)}{r(x)}-1\right)=\frac{1}{p} .
\end{aligned}
$$

By part (a), $\left\{\sum_{k=0}^{n} a_{k}\right\} \in R_{1-\frac{1}{p}}$. Again this follow obviously if $c(0)<\infty$.

Suppose now $\left\{\sum_{k=0}^{n} a_{k}\right\} \in R_{1-\frac{1}{p}}$ with $1<p<\infty$. Using the above identity and part (a) we obtain

Hence

$$
\lim _{x \rightarrow 0}(x b(x)) /\left(\int_{x}^{\eta} b(y) d y\right)=p-1 .
$$

$$
b(x)=x^{-p} L(x) \quad \text { with } L \in R_{0}\left(0^{+}\right) \quad \text { by Lamperti's criterion. }
$$

Since $b^{\prime}(x)=-\frac{1}{r(x)}$ is also increasing the criterion yields

$$
\frac{1}{r(x)} \sim p x^{-1-p} L(x)(x \rightarrow 0),
$$

completing the proof of the lemma. 
Proof of Theorem 2. Let $A$ be in $E(T)$. We have to show that $\left\{\mu\left(A_{n}\right)\right\} \in R_{1-\frac{1}{p}}$. By Lemma 5 in [14],

$$
\mu\left(A_{n}\right) \sim \sum_{j \in J} c_{j}\left(\sum_{k=0}^{n}\left(f_{j}^{k}(1)-x_{j}\right)\right)+\sum_{k=0}^{n}\left(x_{j}-f_{j}^{k}(0)\right)
$$

with positive constants $c_{j}$.

Let $j \in J$ be fixed, and $x_{j}<1$. Since $T x-x \in R_{1+p_{j}^{+}}\left(x_{j}^{+}\right)$and $T^{\prime}$ is monotone in a right neighbourhood of $x_{j}$,

$$
\lim _{x \rightarrow x_{j}^{+}} \frac{\left(x-x_{j}\right)\left(T^{\prime}(x)-1\right)}{T x-x}=1+p_{j}^{+} .
$$

Taking into account that $T^{\prime}\left(x_{j}\right)=1$ this implies

$$
\lim _{x \rightarrow x_{j}^{+}} \frac{\left(x-x_{j}\right)\left(1-f_{j}^{\prime}(x)\right)}{x-f_{j}(x)}=1+p_{j}^{+},
$$

i.e. $x-f_{j}(x) \in R_{1+p_{j}^{+}}\left(x_{j}^{+}\right)$. Applying part (b) of Lemma 3 to the function $f(x)=$ $f_{j}\left(x_{j}+x\right)-x_{j}$ we see that the sequence $\left\{\sum_{k=0}^{n}\left(f_{j}^{k}(1)-x_{j}\right)\right\}$ is regularly varying with index $1-\frac{1}{p_{j}^{+}}$. The same reasoning applies to the left hand side of $x_{j}$ (if $\left.x_{j}>0\right)$. Therefore $\left\{\mu\left(A_{n}\right)\right\} \in R_{1-\frac{1}{p}}$, where $p$ is the maximum of all numbers $p_{j}^{+}, p_{j}^{-}, j \in J$.

Remarks. (i) Concerning the necessity of condition 5, we refer to the special case where $x_{0}=0$ is the only indifferent fixed point of $T$. According to the second statement in part (b) of Lemma 3, in this case the condition

$$
\left\{\mu\left(A_{n}\right)\right\} \in R_{1-\alpha}(A \in E(T)) \text { with } 0<\alpha<1
$$

implies condition 5. (Of course, in the general case no inference is possible on fixed points which do not contribute essentially to the wandering rate.)

(ii) Seeing condition 5 in connection with the asymptotic behaviour of areas determined by the graph of the invariant density results in a stronger geometric interpretation of both the index $\alpha$ and the wandering rate.

Let $T$ be of the special form referred to in (i). Let $F(z)$ denote the area under the graph of the truncated invariant density $\min \{h, z\}$, i.e. $F(z)=\int_{0}^{z} \lambda(h>y) d y$, $z>0$. Let further $\rho(z)$ be the ratio

$$
\rho(z)=\frac{z \lambda(h>z)}{F(z)}
$$

and let $A$ be in $E(T)$ and $0<\alpha<1$. Then we have the following observations.

1. The wandering rate $\left\{\mu\left(A_{n}\right)\right\}$ is regularly varying with index $1-\alpha$, if and only if $\lim _{z \rightarrow \infty} \rho(z)=1-\alpha$.

2. If the condition is satisfied,

$$
\mu\left(A_{n}\right) \sim c . F(n) \quad(n \rightarrow \infty)
$$

for some constant $c>0$.

Recall that

$$
h(x)=h_{0}(x) \cdot g(x) \text { with } g(x)=\frac{x}{x-f_{0}(x)},
$$


where $h_{0}$ is continuous and positive on $[0,1]$. Hence the assertions are not affected, if we replace $h$ by the function $g$.

To prove them, let $F(z)$ and $\rho(z)$ be defined with respect to $g$, and let $r(x)=$ $x-f_{0}(x)$ in accordance with our previous notation. Since $g$ is decreasing,

$$
F(g(x))=x g(x)+\int_{x}^{1} g(y) d y, \quad 0<x \leq 1,
$$

and hence

$$
\lim _{z \rightarrow \infty} \rho(z)=1-\alpha, \text { if and only if } \lim _{x \rightarrow \infty}(x g(x)) /\left(\int_{x}^{1} g(y) d y\right)=\frac{1}{\alpha}-1 .
$$

By Lamperti's criterion, the right hand side implies $g \in R_{-\frac{1}{\alpha}}\left(0^{+}\right)$resp. $r \in$ $R_{1+\frac{1}{\alpha}}\left(0^{+}\right)$.

Suppose conversely that $r \in R_{1+p}\left(0^{+}\right)$, i.e. $\lim _{x \rightarrow \infty} \frac{x r^{\prime}(x)}{r(x)}=1+p$, where $1<$ $p<\infty$. Using l'Hospital's rule we obtain

$$
\lim _{x \rightarrow 0}(x g(x)) /\left(\int_{x}^{1} g(y) d y\right)=p-1 .
$$

This completes the proof af the first assertion and shows that

$$
F(g(x)) \sim p \int_{x}^{1} g(y) d y(x \rightarrow 0) .
$$

For $A \in E(T)$,

$$
\begin{aligned}
\mu\left(A_{n}\right) & \sim \mu\left(\left[a_{n}, 1\right]\right) \sim h_{0}(0) \int_{a_{n}}^{1} g(y) d y \\
& \sim\left(h_{0}(0) / p\right) F\left(g\left(a_{n}\right)\right), \text { where } a_{n}=f_{0}^{n}(1), n \geq 0 .
\end{aligned}
$$

On the other hand,

$$
g(x) \sim p \int_{x}^{1} \frac{d y}{r(y)} \quad(x \rightarrow 0),
$$

as is also seen most readily by means of l'Hospital's rule, and therefore

$$
g\left(a_{n}\right) \sim p \int_{a_{n}}^{1} \frac{d y}{r(y)} \sim p n \quad(n \rightarrow \infty) .
$$

Since $F(z)$ is regularly varying with index $1-\frac{1}{p}$ for $z \rightarrow \infty$,

$$
F\left(g\left(a_{n}\right)\right) \sim p^{1-\frac{1}{p}} F(n) \quad(n \rightarrow \infty),
$$

completing also the proof of the second assertion.

Acknowledgement. The author would like to thank R. Zweimüller for valuable comments on this paper, in particular for contributing to the remark at the end of section 3 .

\section{REFERENCES}

[1] J. Aaronson, The asymptotic distributional behaviour of transformations preserving infinite measures, J. Analyse Math. 39 (1981), 203-234 MR 82m:28030

[2] J. Aaronson, Random f-expansions, Ann. Probab. 14 (1986), 1037-1057 MR 87k:60057

[3] J. Aaronson, An introduction to infinite ergodic theory, AMS, 1997 CMP 97:13

[4] N.H. Bingham, C.M. Goldie, J.L. Teugels, Regular variation, Cambridge University Press, Cambridge 1987 MR 88i:26004 
[5] D.A. Darling and M. Kac, On occupation times for Markoff processes, Trans. Amer. Math. Soc. 84 (1957), 444-458 MR 18:832a

[6] E.B. Dynkin, Some limit theorems for sums of independent random variables with infinite mathematical expectations, Selected Transl. in Math. Statist. and Probability 1 (1961), 171189 MR 22:7164

[7] W. Feller, An introduction to probability theory and its applications, Vol. II. John Wiley \& Sons, New York 1971 MR 42:5292

[8] J. Lamperti, An occupation time theorem for a class of stochastic processes, Trans. Amer. Math. Soc. 88 (1958), 380-387 MR 20:1372

[9] J. Lamperti, Some limit theorems for stochastic processes, J. Math. Mech. 7 (1958), 433-448 MR 20:4888

[10] P. Manneville, Intermittency, self-similarity and $1 / f$ spectrum in dissipative dynamical systems, J. Physique 41 (1980), 1235-1243 MR 82e:58065

[11] F. Schweiger, Ergodic theory of fibered systems and metric number theory, Clarendon Press, Oxford 1995 MR 97h:11083

[12] R.S. Slack, Further notes on branching processes with mean one, Z. Wahrscheinlichkeitstheorie verw. Geb. 25 (1972), 31-38 MR 48:9871

[13] M. Thaler, Estimates of the invariant densities of endomorphisms with indifferent fixed points, Isr. J. Math. 37 (1980), 303-314 MR 82f:28021

[14] M. Thaler, Transformations on [0, 1] with infinite invariant measures, Isr. J. Math. 46 (1983), 67-96 MR 85g:28020

[15] M. Thaler and C. Reichsöllner, Arc sine type limit laws for interval mappings, Manuscript, Salzburg 1986

[16] M. Thaler, A limit theorem for the Perron-Frobenius operator of transformations on [0,1] with indifferent fixed points, Isr. J. Math. 91 (1995), 111-127 MR 96i:28020

[17] M. Thaler, The invariant densities for maps modeling intermittency, J. Statist. Phys. 79 (1995), 739-741 MR 96a:58119

[18] R. Zweimüller, Probabilistic properties of dynamical systems with infinite invariant measure, Diplomarbeit, Salzburg 1995

Institute of Mathematics, University of Salzburg, Hellbrunnerstrasse 34, A-5020 Salzburg, Austria

E-mail address: Maximilian.Thaler@sbg.ac.at 